\title{
Le réseau d'enseignement public bas-canadien, 1841-1867 : une institution de l’État libéral
}

\section{Jean-Pierre Charland}

Volume 40, numéro 4, printemps 1987

URI : https://id.erudit.org/iderudit/304490ar

DOI : https://doi.org/10.7202/304490ar

Aller au sommaire du numéro

Éditeur(s)

Institut d'histoire de l'Amérique française

ISSN

0035-2357 (imprimé)

1492-1383 (numérique)

Découvrir la revue

Citer cet article

Charland, J.-P. (1987). Le réseau d'enseignement public bas-canadien, 1841-1867: une institution de l'État libéral. Revue d'histoire de l'Amérique française, 40(4), 505-535. https://doi.org/10.7202/304490ar
Résumé de l'article

Les quelques années qui suivent l'union des Canadas connaissent en quelque sorte le dernier effort des colonies nord-américaines pour l'obtention du gouvernement responsable. Quoique l'on ait surtout écrit sur les incidences nationales de cette nouvelle constitution, celle-ci témoigne aussi du triomphe d'un certain nombre d'idées libérales. Les artisans du système scolaire du Canada-Est vont faire du réseau d'enseignement une institution ayant pour but de faire partager l'idéologie des réformistes au plus grand nombre. Aussi pouvons-nous trouver, dans les écrits des surintendants et des inspecteurs d'écoles, des actes de foi libéraux sans cesse réitérés ; de même, au niveau des structures éducatives, nous retrouvons des institutions qui, tout en laissant une certaine autonomie aux populations locales (grâce aux commissions scolaires), permettent une centralisation lente des pouvoirs. Ce centralisme peut paraître contredire l'esprit et la lettre de l'idéologie libérale, mais il nous semble plutôt que, face aux contestations dont le réseau mis en place était l'objet (la guerre des " éteignoirs ", bien sûr, mais aussi la gourmandise croissante du clergé face à ce réseau), les autorités, surintendant et inspecteurs en tête, mais aussi le Conseil de l'Instruction publique, pragmatiques, se donnaient ainsi les pouvoirs requis pour infléchir les contenus d'enseignement et assurer que tout le réseau continuât d'épouser les idéaux libéraux. Ce n'est qu'après 1867 que le clergé allait investir le réseau scolaire par les brèches qu'il avait pu s'y ménager...
Tous droits réservés @ Institut d'histoire de l'Amérique française, 1987
Ce document est protégé par la loi sur le droit d'auteur. L’utilisation des services d'Érudit (y compris la reproduction) est assujettie à sa politique d'utilisation que vous pouvez consulter en ligne.

https://apropos.erudit.org/fr/usagers/politique-dutilisation/ 


\title{
LE RÉSEAU D'ENSEIGNEMENT PUBLIC BAS- CANADIEN, 1841-1867: UNE INSTITUTION DE L'ÉTAT LIBÉRAL ${ }^{1}$
}

\author{
JEAN-PIERRE CHARLAND \\ Département d' histoire \\ Université d'Ottawa
}

\begin{abstract}
RÉSUMÉ
Les quelques années qui suivent l'union des Canadas connaissent en quelque sorte le dernier effort des colonies nord-américaines pour l'obtention du gouvernement responsable. Quoique l'on ait surtout écrit sur les incidences nationales de cette nouvelle constitution, celle-ci témoigne aussi du triomphe d'un certain nombre d'idées libérales. Les artisans du système scolaire du CanadaEst vont faire du réseau d'enseignement une institution ayant pour but de faire partager l'idéologie des réformistes au plus grand nombre. Aussi pouvons-nous trouver, dans les écrits des surintendants et des inspecteurs d'écoles, des actes de foi libéraux sans cesse réitérés; de même, au niveau des structures éducatives, nous retrouvons des institutions qui, tout en laissant une certaine autonomie aux populations locales (grâce aux commissions scolaires), permettent une centralisation lente des pouvoirs. Ce centralisme peut paraître contredire l'esprit et la lettre de l'idéologie libérale, mais il nous semble plutôt que, face aux contestations dont le réseau mis en place était l'objet (la guerre des «éteignoirs», bien sûr, mais aussi la gourmandise croissante du clergé face à ce réseau), les autorités, surintendant et inspecteurs en tête, mais aussi le Conseil de l'Instruction publique, pragmatiques, se donnaient ainsi les pouvoirs requis pour infléchir les contenus d'enseignement et assurer que tout le réseau continuât d'épouser les idéaux libéraux. Ce n'est qu'après 1867 que le clergé allait investir le réseau scolaire par les brèches qu'il avait pu s'y ménager...
\end{abstract}

\section{ABSTRACT}

The years which followed the Union of the Canadas witnessed the final efforts of the British North American colonies to secure responsible government. While the national impact of the new constitutional arrangements has been extensively described, they were also the product of liberal ideas. The architects of the Canada East school system made it into an institution designed to promote reformist ideology. Thus we find, in the writings of school inspectors and superintendants of education, numerous professions of faith in liberalism; as well, the education system itself, while leaving a degree of autonomy to the local population through the school boards, was geared towards a steady centralization of power. While centralization may appear in contradiction with the spirit and the letter of liberalism, educational authorities (the Superintendant, the inspectors, and the Conseil de l'instruction publique), faced with the challlenges to the system from the "candle snuffers" and threatened by the clergy's unrelenting attempts at control, gave themselves enough power to shape educational contents and to ensure that the school system continue to promote liberal ideas. It is only after 1867 that the clergy was able to take over the school system through the doors it had managed to open in the previous period.

1 Ce texte reprend et complète une communication présentée au Congrès de l'Institut d'histoire de l'Amérique française, à Montréal, en octobre 1985, et intitulée «Le développement de l'État québécois et l'enjeu éducatif». 
Dans un article déjà ancien, Nadia Fahmy-Eid établissait avec raison que dans sa lutte pour accaparer le système scolaire, la petite-bourgeoisie québécoise, à cause de sa faiblesse économique et politique, avait dû composer avec l'Église catholique ${ }^{2}$. Ce compromis était d'autant plus facile que le Parti conservateur avait reçu l'appui des clercs pour se maintenir au pouvoir pendant un demi-siècle. L'auteure, à la suite d'historiens de l'éducation comme Groulx, Filteau et même Audet ${ }^{3}$, paraissait attribuer uniformément le même caractère clérical et conservateur, au système scolaire de 1840 à 1867, qu'à celui de 1875, et faisait la liste de «caractères confessionnels» qui me semblent avoir été mineurs avant la Confédération ${ }^{4}$. Je ne peux la suivre jusque-là. Au contraire, je me propose de montrer combien le réseau scolaire qui s'élaborait entre 1841 et 1861 traduisait les idéaux libéraux d'accessibilité, de tolérance religieuse et de développement économique, et que ces idéaux étaient présents dans l'esprit de ceux qui ont élaboré le réseau d'enseignement (je reviendrai plus loin sur ces «idéaux»). L'État d'ailleurs s'était donné les pouvoirs requis pour s'assurer un contrôle suffisant des institutions scolaires et avait pu ainsi faire en sorte que le réseau atteindrait les objectifs qu'il avait fixés.

Bien sûr cela ne se fit pas sans concessions à l'Église, mais le département de l'Instruction publique se réserva l'essentiel des responsabilités. Et si le groupe au pouvoir ne prononçait pas les discours

2 Nadia F.-Eid, «Éducation et classes sociales: analyse de l'idéologie conservatrice-cléricale et petite-bourgeoise au Québec au milieu du 19e siècle», Revue d'histoire de l'Amérique française, 32,2 (septembre 1978): 159-179. Elle ne se distingue pas vraiment de Fernand Ouellet, «L'enseignement primaire: responsabilité des Églises ou de l'État (1801-1836)», Recherches sociographiques (1961): 171-187, quand elle souligne les aspects conservateurs de ce système scolaire.

Lionel Groulx, L'enseignement français au Canada. I - Dans le Québec; Gérard Filteau, Les constantes historiques de notre système scolaire, et Le système scolaire de la province de Québec: historique, législation et règlements; Louis-Philippe Audet, Histoire de l'enseignement au Québec, 1608-1971. Ce n'est qu'à propos de cette identification des aspects cléricaux et conservateurs du réseau scolaire que nous pouvons mettre ces auteurs ensemble. Bien sûr, Nadia Fahmy-Eid, et Louis-Philippe Audet dans une plus faible mesure, ne voient pas comme une bénédiction ces caractères cléricaux, contrairement à Groulx et à Filteau.

4 Tout est sans doute question de perception: il me semble pourtant que le droit de dissidence et la confessionnalité des écoles de Montréal et de Québec, tout comme le choix des manuels servant à l'enseignement religieux ou moral par les autorités religieuses, et la possibilité pour des membres du clergé de siéger sur les commissions scolaires, même sans la qualification foncière requise pour les autres commissaires (tous des avantages accordés par la Chambre d'assemblée, et pouvant au moins théoriquement être révoqués par elle), s'ils ouvraient une faille dont l'Église catholique allait savoir profiter, ne rendaient pas le réseau scolaire bas-canadien confessionnel pour autant. Je ne voudrais pas m'étendre sur les notions de droit ici, mais le lecteur pourrait avec profit consulter Guy Houle (Le cadre juridique de l'administration scolaire locale au Québec, Annexe au rapport de la Commission royale d'enquête sur l'enseignement dans la province de Québec, 1966), selon lequel seules les commissions scolaires dissidentes et celles de Montréal et de Québec avaient, légalement, un caractère confessionnel (et à ce titre jouissaient de la protection constitutionnelle). Bien plus, les sentences, lors des nombreux procès qui ont opposé les commissions scolaires au ministère de l'Éducation, ont toujours confirmé ce fait. Et un juge au moins est allé jusqu'à préciser qu'à Montréal et à Québec, seules les écoles situées à l'intérieur des frontières de 1867 des commissions scolaires étaient confessionnelles de droit! Quant à la confessionnalité de fait, il faudrait des monographies nombreuses pour évaluer la situation avant 1867. 
libéraux les plus tonitruants (ils étaient plutôt le fait des «rouges»), cela ne prouve en rien que les artisans du système scolaire comme les législateurs n'aient pas adhéré à la même idéologie: tout est question de nuances ${ }^{5}$. Ce n'est qu'après 1867 que l'Église accapara la direction du système d'enseignement et remplaça largement les objectifs d'un État libéral par les siens propres.

Pour mieux démontrer le caractère conservateur du régime scolaire, l'auteure rappelait encore la brièveté du cursus scolaire au BasCanada, insistant sur les trois ou quatre années du programme établi par Mgr Langevin en 1872, dont nous savons pourtant, notamment grâce à L.-P. Audet, qu'il s'agissait de cycles de formation, mais qu'elle présentait comme des années de «calendrier». Elle relevait aussi le caractère peu démocratique du réseau scolaire, invoquant les faibles résultats de l'effort de scolarisation des habitants. Mais étaient-ils si faibles? Vers 1860 , on peut croire qu'un peu plus de la moitié de la population du Québec d'âge scolaire fréquentait l'école, contre $70 \%$ en Ontario $^{6}$. La proportion devait être aussi plus forte dans certains États de la Nouvelle-Angleterre. Mais Chauveau, de retour de sa tournée européenne en 1867, affirmait que nous n'avions rien à envier à l'Irlande, l'Angleterre, l'Écosse, la France, la Belgique et une partie de l'Allemagne. Compte tenu de la pauvreté du Québec, qu'illustrait déjà le flot migratoire, la province avait consenti un effort immense pour améliorer la scolarisation de la population, en rêvant que cela permettrait l'enrichissement de la communauté. Et encore là, il faudrait vérifier soigneusement quel était ce retard sur l'Ontario, en tenant compte d'un absentéisme parfois effroyable des élèves inscrits aux écoles de cette province. On le voit, une fois encore je ne peux suivre Nadia Fahmy-Eid, ni d'ailleurs quand elle semblait invoquer le fait que ce réseau scolaire devait «reproduire les rapports de production» pour illustrer son caractère conservateur. Entre le conservatisme à la sauce «cléricale» et une école de conscientisation, de contestation, il y a pourtant toutes les nuances libérales, qui elles aussi ne voudront pas que l'école ne bouleverse l'ordre social.

En fait, Fahmy-Eid témoignait du ressac de la Révolution tranquille. Autant les auteurs cléricaux avaient cherché à plonger les racines du contrôle clérical sur l'école dans le passé le plus lointain, et à tailler à l'Église une place de héros en affirmant qu'elle avait réussi à détour-

5 Que les partis s'appellent conservateur et radical ne signifie pas qu'ils ne pouvaient adopter, globalement, la même idéologie. Même en Angleterre les conservateurs finirent par adopter des mesures «réformistes». Dans le système parlementaire britannique, il n'est guère surprenant de voir les deux partis habituels s'inspirer de la même idéologie et ne se démarquer l'un de l'autre que par de simples nuances.

6 Lionel Groulx, op. cit. Ces chiffres étaient repris par L.-P. Audet dans «Le premier ministère de l'Instruction publique au Québec, 1867-1875», RHAF, 22,2 (septembre 1968): 171222. 
ner les funestes desseins scolaires (et nationaux) des Buller et Durham et à sauver la nation (cela après les aventures néfastes des élites laïques, en 1837-1838), en redonnant au peuple ses écoles grâce aux commissions scolaires, autant ceux des années 1960 et 1970 ont été sévères envers le rôle du clergé catholique dans l'histoire québécoise, lui attribuant largement la responsabilité des «retards» que la province avait accumulés. Ce faisant, ces sociologues ou ces historiens ont parfois noirci le tableau, amplifiant l'emprise cléricale sur la société pour mieux la condamner ensuite. Aussi tous les «symptômes» d'un contrôle clérical sur le réseau scolaire et les mauvais effets attribués à celui-ci, notamment le conservatisme, la résistance aux idées modernes, retenaient-ils plus facilement l'attention que les éléments qui auraient pu témoigner du contraire. Bien plus, il était difficile, dans le contexte de ces années, de prêter quelques qualités au régime scolaire établi dans la foulée du rapport Durham. Reprocher au clergé d'avoir retardé le développement du Québec tout en lui reconnaissant le mérite d'avoir largement aidé à sauver la nation dans ces années difficiles permettait de satisfaire à la fois la nouvelle génération volontiers iconoclaste, mais pour qui une teinte nationale pouvait tout faire excuser, et une vieille garde qui s'incrustait? .

Les objectifs du présent article sont plutôt modestes. Le lecteur trouvera d'abord une brève description des objectifs libéraux confiés au réseau scolaire par ses maîtres d'oeuvre, surintendants ou inspecteurs. Ceux-ci identifiés, nous verrons par le biais d'un examen de la législation scolaire les moyens qu'ils se sont donnés pour les atteindre. En effet, si j'insiste sur l'étendue du contrôle que s'attribuait le département de l'Instruction publique sur le réseau scolaire, c'est parce qu'il me semble que ses dirigeants se donnaient là le moyen de répandre, dans la population, ces idéaux libéraux. Bien sûr, je ne suis pas sans savoir que pendant les décennies qui nous intéressent ici le clergé a pu placer ses pions, créant ou important des congrégations religieuses, menant de grandes campagnes publicitaires avec parfois l'aide de «vedettes» étrangères, et établissant des liens suivis avec le parti au pouvoir. Il a même pu s'assurer des brèches dans l'édifice scolaire qui lui ont permis de l'investir totalement en 1875. Un prochain article devrait permettre d'illustrer combien le surintendant et ses employés pouvaient, dans le fonctionnement quotidien des écoles, en assurer le caractère libéral.

7 Une publication récente nous apprenait que Mgr Paul-Émile Léger acceptait encore assez mal que le bill 60 , qui créait le ministère de l'Éducation, n'ait pas reconnu, dans son préambule, la dette de la communauté québécoise à l'égard de l'Église à ce sujet. Micheline Lachance, Dans la tempête. Le cardinal Léger et la Révolution tranquille (Montréal, Éditions de l'Homme, 1986). 


\section{LES OBJECTIFS LIBÉRAUX DU RÉSEAU SCOLAIRE PUBLIC}

Au moment où l'Angleterre imposait à sa colonie une nouvelle constitution, Louis-Hippolyte La Fontaine et Augustin-Norbert Morin, chefs du Parti réformiste, qui regroupait les éléments modérés du Parti patriote disparu avec l'échec des insurrections, s'unirent aux dirigeants libéraux du Haut-Canada, Hincks et Baldwin. Ils décidèrent de faire front commun dans le but d'obtenir la responsabilité ministérielle. La Fontaine fit le pari que si les coloniaux obtenaient la direction de leur gouvernement, les francophones auraient en chambre un poids tel qu'aucun parti ne pourrait se maintenir au pouvoir sans respecter leurs intérêts nationaux (si ceux-ci se limitaient à la stricte survie culturelle, bien sûr). L'histoire lui donna raison. L'espoir d'obtenir le gouvernement responsable comportait aussi ses exigences éducatives propres. Le Parti patriote avait eu besoin des écoles de syndics pour façonner la nation, assurer sa cohésion et la conduire à l'indépendance ${ }^{8}$. Le Parti réformiste, lui, voulait que l'école prépare les gens à une quasi-souveraineté. Le peuple devait pouvoir s'habituer à la vie démocratique, élire des gens capables de conduire les destinées de la colonie, assurer la prospérité du territoire. On ne pense pas assez que l'électeur créait le lecteur, que l'extension du suffrage à de nouvelles couches sociales accompagnait largement celle de l'alphabétisation, car la vie parlementaire exigeait que la population pût s'informer.

Quand on connaît les centaines d'ouvrages qui concernent l'idéologie libérale, on ne peut que mesurer tous les risques qu'il y a à essayer de donner, en quelques lignes, une idée exacte des traits libéraux que je vais chercher dans le système scolaire québécois ${ }^{9}$. Surtout que le libéralisme est à la fois un système philosophique, un système économico-social concret et une école d'économie politique. Au niveau philosophique, il s'appuie sur la conviction de l'égalité des hommes entre eux, de l'existence de droits individuels irrépressibles et d'une tendance à rechercher le bonheur que les pouvoirs publics devraient favoriser, notamment en garantissant le droit de propriété de chacun. Ces principes pourront prendre une forme concrète dans la recherche spontanée de l'intérêt. En effet, en ce qui concerne l'économie politique, on établit que la recherche de la richesse par les individus, dans le cadre du marché régi par le libre-échange, par la concurrence, va conduire néces-

\footnotetext{
8 Fernand Ouellet, loc. cit.

9 Beaucoup trouveront cette «description» trop brève. Le lecteur pourra se référer aux classiques Smith, Ricardo, Bentham, Mill, aux ouvrages d'André Vachet, L'idéologie libérale (Paris, Anthropos, 1970), d'Eric J. Evans, The Forging of Modern State. Early Industrial Britain, 1783-1870 (London and New York, Longman, c1983), de Serge-Christophe Kolm, Le contrat social libéral: philosophie et pratique du libéralisme (Paris, Presses universitaires de France, 1985), de Didier Deleule, Hume et la naissance du libéralisme économique (Paris, Aubier Montaigne, c1979), d'Harry K. Girvetz, The Evolution of Liberalism (London, Collier Books, c1969), entre autres.
} 
sairement et aux moindres frais à un état d'équilibre entre la production et la consommation, à la satisfaction des attentes des personnes. En fait, ces principes s'opposent à toute intervention de l'État ou de groupes constitués dans les échanges économiques ou dans la production. On a la conviction que l'État ne devrait se limiter qu'à assumer un rôle judiciaire (s'assurer du respect des contrats et de la jouissance parfaite de la propriété). Même les premiers économistes reconnaissaient qu'il pouvait y avoir abus, un accaparement de la part des possédants, mais ils croyaient néanmoins que les individus, mûs par la poursuite de leurs intérêts personnels, et le jeu de la concurrence, du marché, allaient conduire à une harmonisation des efforts, et finalement à la satisfaction des aspirations du plus grand nombre, mieux que n'importe quelle intervention d'État. Le pire gouvernement étant celui qui intervient, le meilleur celui qui demeure discret...

Ces principes étaient véhiculés en Angleterre dès la fin du 17e siècle, en même temps qu'au lendemain de la «Glorious Revolution» la bourgeoisie commençait une ascension qui allait la conduire au leadership de la société. Il faut bien replacer dans ce contexte les éléments énumérés ci-dessus. La reconnaissance de l'égalité des individus devant la loi, la recherche du bonheur par chacun, étaient liés aux obstacles que posaient les privilèges conférés à l'aristocratie, à la liberté d'entreprendre de la bourgeoisie. La réticence à voir l'État ou des associations (comme les corporations de métier) intervenir dans la vie économique tenait aux obstacles qu'ils posaient au libre fonctionnement du marché, essentiel à la prospérité. Dans ce contexte, le meilleur État possible était celui qui pouvait garantir la libre jouissance des propriétés et le libre jeu de la concurrence. Notons bien que, au 18e siècle, cela signifiait la fin des obstacles intérieurs aux échanges commerciaux, l'adoption d'un droit qui favoriserait l'établissement de contrats entre individus libres (l'interdiction des associations de travailleurs) et l'apparition d'un État qui serait sensible aux intérêts du commerce et de l'industrie. Rien de mieux alors que la démocratie parlementaire (rappelons que le suffrage était limité aux propriétaires), qui traduirait les intérêts de moyens ou petits entrepreneurs. Bien plus, la responsabilité ministérielle, réclamée par le parti libéral anglais, interprète des intérêts de la bourgeoisie, allait obliger le gouvernement à répondre de ses décisions devant les élus.

L'État allait aussi devoir réaliser cette égalité civile des citoyens. En Grande-Bretagne, peu avant 1830, il reconnaissait les mêmes droits aux membres des diverses confessions religieuses et mettait fin au règne de l'Église d'Angleterre comme religion d'État ${ }^{10}$. De plus, il faut ajou-

10 Dans le Haut-Canada, Ryerson menait une lutte en ce sens, notamment pour que les anglicans ne puissent avoir un monopole de l'enseignement. Rappelons au passage que la montée de la bourgeoisie s'accompagnait d'une méfiance certaine à l'égard des Églises, qui avaient lar- 
ter à la liste des revendications libérales l'adoption du libre-échange. Entre 1830 et 1870 , c'est l'époque du «laisser-faire» et du «laisserpasser». Enfin, formes parlementaires et «dépérissement de l'État»n'ont pas nécessairement prévalu dans tous les pays où l'organisation économique et le cadre légal, eux, étaient libéraux, comme en France ou en Allemagne ${ }^{11}$.

Dans le Bas-Canada, pendant les années qui vont de 1800 à 1875 , l'idéologie libérale avait, bien sûr, pris des formes spécifiques. D'abord à cause de son statut colonial et de sa jeunesse, la capitalisation n'avait encore été que faible: pendant longtemps les développeurs ne purent assurer leurs projets qu'avec l'implication de Britanniques, mais aussi celle de l'État, dont la participation était essentielle dans les grands projets (les canaux d'abord, puis les chemins de fer, et enfin la régulation des cours d'eau qui en permit le harnachement). La spécificité bas-canadienne se trouvait aussi exprimée dans les alignements politiques. Contrairement à l'Ontario, où les entrepreneurs se retrouvaient au sein du Parti réformiste et s'opposaient au «Family Compact» qui freinait le développement capitaliste au Québec, on retrouvait en effet les entrepreneurs, majoritairement de langue anglaise, avec les «bureaucrates», dans ce qu'il était permis d'appeler la «clique du château», alors qu'au sein du Parti patriote, réunies par le projet national, on retrouvait des tendances conservatrices et libérales ${ }^{12}$. Pour moi, cela n'infirme pas ma croyance en un courant libéral qui avait pu devenir dominant chez l'élite politique du Bas-Canada, sous le régime du Canada-Uni ${ }^{13}$; sauf que ce mouvement avait dû prendre des formes particulières. En fait, nous trouvons les inévitables adaptations de la doctrine à la conjoncture, comme partout ailleurs. Après 1841, le Parti conservateur comme le Parti réformiste allaient représenter les intérêts de la bourgeoisie, n'étant éloignés que par des nuances.

Au Bas-Canada, comme J.-B. Meilleur et P.-J.-O. Chauveau l'ont écrit, on en était dans «l'enfance des institutions». Les habitants étaient peu familiers avec les municipalités ou le parlementarisme, et ils s'en défiaient compte tenu des objectifs assimilateurs que Durham, puis

\footnotetext{
gement garanti l'hégémonie de la noblesse, l'ancienne classe dominante. Certains courants, comme I'utilitarisme de Mill et de Bentham, ou l'anticléricalisme du mouvement républicain français, qui ont tant terrorisé le clergé du Québec, étaient aux antipodes de la notion de religion d'État. Enfin, bien que la bourgeoisie ait fini par mesurer tout l'intérêt des valeurs religieuses comme instrument de contrôle social, elle ne verra pas habituellement d'un bon oeil qu'elles s'emmêlent aux lois du marché ou au fonctionnement de l'État. Dans le premier cas, on préfère avoir affaire à des agents économiques, dans le second à des citoyens. Le libéralisme est, en dernière instance, laïque.

11 Cependant, même en Angleterre, entre 1830 et 1870 , l'État a été beaucoup plus interventionniste que ne le veut l'historiographie traditionnelle. F. Bédarida, «L'Angleterre victorienne paradigme du laissez-faire?...», Revue historique, 261,1: 79-98.

A ce sujet, voir Fernand Ouellet, Le Bas-Canada, 1791-1840 (Ottawa, Éditions de l'Université d'Ottawa, 1976).

13 Jacques Monet, La première révolution tranquille. Le nationalisme canadien-français (1837-1850) (Montréal, Fides, c1981).
} 
Sydenham leur avaient confiés. Dans ce contexte, malgré la doctrine, la centralisation, l'intervention de l'État allait chercher à répandre ces idéaux libéraux, même si les moyens nous paraissent avoir été peu compatibles avec la théorie. Nous verrons, lorsque nous examinerons la loi de 1841 combien la centralisation des pouvoirs en ce qui concerne l'éducation, pouvait tout de même être libérale. Mais, même quand les États commencèrent à intervenir massivement (en Angleterre on parle, pour la période d'après 1870 , d'époque «collectiviste»), cela ne voulut pas nécessairement dire que l'on reniait les idéaux libéraux ${ }^{14}$ : la concentration du capital, la paupérisation des masses liée à l'industrialisation, la présence d'associations ouvrières exigeaient une intervention accrue pour limiter les excès du régime, de façon à ce que la libre entreprise ne soit pas remise en cause...

Tous ceux qui ont une certaine culture historique ont appris, au Québec, à honnir Durham pour ses remarques sur l'histoire et la culture des francophones, et la «solution finale» qu'il proposait au problème national. Et tous semblent avoir oublié que c'était aussi un libéral qui condamna le «Family Compact» ontarien et conseilla au gouvernement britannique de concéder le gouvernement responsable à ses colonies d'Amérique. Les institutions de 1840 portaient les espoirs, sinon les germes, nés de ces recommandations. Ce fut grâce à ces espoirs que les groupes modérés des Partis réformistes et patriote purent se regrouper et poursuivre un objectif commun. Et ce fut dans ce contexte que Jean-Baptiste Meilleur, surintendant de l'Éducation pour le Bas-Canada, établit les objectifs libéraux du réseau scolaire, et voulut que l'État jouisse à ce sujet des pouvoirs nécessaires pour les faire valoir, comme en témoigne cette déclaration de 1848:

Il est admis par tous les philanthropes qu'il faut faire le bien aux hommes souvent malgré eux; telle est l'obligation des pères de famille vis-à-vis de leurs enfants, telle est aussi, à plus forte raison, l'obligation des gouvernements envers les pères de famille et envers leurs enfants. Or, l'instruction est le plus grand bien que les pères de famille et que les gouvernements puissent faire aux enfants. ${ }^{15}$

Il écrivait un peu plus tard pour renchérir sur l'importance de cette mission:

L'histoire et l'expérience, d'accord avec ce principe (d'éduquer la jeunesse), nous montrent que les pays dont les habitants sont à l'intérieur les plus forts, les plus prospères et les plus heureux, dont le commerce social est le plus agréable, et qui sont les plus

14 Sur ce phénomène au Canada, on pourra consulter Ramsay Cook, The Regenerators. Social Criticism in Late Victorian English Canada (Toronto, Buffalo and London, University of Toronto Press, c1985).

15 Jean-Baptiste Meilleur, «Rapport du surintendant de l'Education pour le Bas-Canada pour l'année 1847», dans Journaux de l'Assemblée, 1848. 
respectés et les plus influents au dehors, sont ceux qui sont les plus généralement instruits. ${ }^{16}$

Bien sûr, on admettait sans peine que les parents soient concernés au premier chef par l'éducation de leurs enfants. Mais cela ne voulait pas dire qu'on leur laissait une entière liberté. Il allait de soi que les bons parents faisaient les sacrifices nécessaires à l'éducation de leurs enfants, et, si les mauvais parents n'assumaient pas leurs responsabilités, l'État devait prendre le relais, passer à la coercition, affirmait encore Meilleur:

Ainsi, lorsque pour l'objet de l'éducation, le gouvernement ne peut obtenir le bon vouloir et la coopération volontaire des parents et des pères de famille, les mesures de rigueur, basées sur la raison et sur des principes de justice, sont justifiables aux yeux de la philanthropie, de la religion et d'une saine politique. C'est alors que tout bon gouvernement, consultant moins les préjugés et les préventions que les besoins et l'intérêt bien entendu de la société, adopte sans hésitation les moyens pratiques et même les mesures de rigueur dont les circonstances rendent l'usage nécessaire, et que, malgré l'indifférence ou l'opposition du peuple, il procure aussi forcément à ses enfants le bienfait de l'éducation. ${ }^{17}$

Car si les gouvernants jugeaient bon d'éduquer le peuple (la confusion entre les mots instruction et éducation est ici significative), il n'était pas évident que tous entendaient se faire éduquer... surtout quand il fallait aborder la question du financement d'un système scolaire universellement accessible. On connaît les charivaris qui allaient accueillir les taxes obligatoires en 1846, que l'histoire a retenus, sous le nom de "guerre des éteignoirs». Dans ce contexte de désordres, le système scolaire était jugé d'autant plus essentiel à la vie démocratique. L'inspecteur Bardy, qui oeuvrait dans la région de Québec, expliquait à ce sujet:

Mais là où les électeurs ne sont pas juges éclairés sur les choix qu'ils doivent faire, l'élection n'exerce plus son droit, l'intrigue sait l'usurper, l'action de la cabale, de la partialité paralyse le régime municipal, si bien qu'enfin, sous l'empire des formes et des règles parlementaires, ce sont le monopole et souvent l'ignorance qui président à la confection des lois. Un état, par exemple, ne produira d'hommes capables de le gouverner, qu'alors que l'instruction, généralement répandue, aura si largement fondé cette hiérarchie des intelligences, que chaque unité municipale, chaque unité électorale, posséderont et des administrateurs au niveau des

16 Jean-Baptiste Meilleur, «Rapport du surintendant de l'Education pour le Bas-Canada pour l'année 1849», dans Journaux de l'Assemblée, 1850. «Circulaire no 12 aux commissaires et aux instituteurs».

17 Jean-Baptiste Meilleur, «Rapport du surintendant de l'Education pour le Bas-Canada pour les années 1842-1843», dans Journaux de l'Assemblée, 1843. 
besoins de la société à satisfaire, et des mandataires en état de faire co-ordonner avec l'intérêt général celui de la localité ou de l'industrie qu'ils représenteront. ${ }^{18}$

Et dans la mesure où l'éducation servait les intérêts de tous, en formant chacun aux institutions parlementaires mais aussi en rendant chacun plus apte à tirer son épingle du jeu au niveau économique, il allait de soi que tous devaient payer pour maintenir le réseau de formation. Bien plus, la contribution allait être proportionnelle aux moyens des individus:

Le système de contribution forcée, pour l'instruction des enfants, en proportion des moyens des parents, est celui qui convient également le mieux aux intérêts bien entendus du pauvre et du riche; car il n'exige du pauvre que ce qu'il peut fournir pour cette fin, et il a l'effet d'obliger le riche à contribuer davantage pour l'instruction des enfants du pauvre, et à améliorer ainsi l'état général de la société, en faisant sortir les enfants du pauvre de la condition d'indigence où ils sont, et où, sans l'instruction, ils demeureraient nécessairement, par suite de leur ignorance et de leur incapacité.

Le riche, par sa contribution pour l'instruction des enfants du pauvre, les fait sortir de cette condition humiliante, et, en les émancipant, le riche s'émancipe lui-même de l'obligation de subvenir à leurs besoins sous forme d'aumônes réitérées, souvent insuffisantes, mais dont le montant, converti en moyens d'instruction, produirait une aisance générale. L'état de la société est ainsi amélioré par l'instruction également répartie, à un degré suffisant, pour faire de tous les enfants des sujets capables, utiles et honorables; des piliers, des soutiens de la société, au lieu d'en être le fardeau, le ver rongeur, et souvent même le fléau, la honte et le désespoir.

Avec l'instruction, aucun n'est à charge à ses parents, à ses amis, à ses voisins, ni comme nécessiteux, ni comme déprédateur; et le riche, vivant ainsi au milieu de citoyens, de voisins prospères et heureux, n'a rien à craindre, rien à payer, si ce n'est pour atteindre le but de la loi, dans lequel chacun trouve son avantage. ${ }^{19}$

C'est toute la notion de l'intérêt public qui était exprimée là... comme celle de la responsabilité publique. Et tout cela n'entrait pas en contradiction avec le «laisser-faire». Au contraire, le système scolaire était présenté comme l'un des instruments de l'État pour assurer la libre-jouissance de la propriété par les individus. L'intervention de l'État dans les affaires éducatives venait compléter son intervention au niveau

18 Pierre-Joseph-Olivier Chauveau, «Rapport du surintendant de l'Instruction publique pour le Bas-Canada pour l'année 1855», dans Journaux de l'Assemblée, 1856. Rapport de l'inspecteur Bardy. 1853.

Jean-Baptiste Meilleur, «Education Bas-Canada, 1853», dans Journaux de l'Assemblée, 
de la justice, pour créer les conditions d'existence du régime libéral. Le surintendant Meilleur répéta sans cesse cet acte de foi, surtout que pendant plusieurs années il dut faire face à la contestation des taxes scolaires. Il croyait que l'instruction permettrait d'enrayer la misère, la pauvreté, car le savoir rendrait chacun plus apte à se débrouiller, à poursuivre ce «bonheur», à chercher son intérêt. Elle concrétiserait cette liberté d'entreprendre en donnant aux personnes les moyens intellectuels de le faire, et l'intérêt général (l'abondance pour tous) viendrait de ce que chacun pourrait satisfaire ses besoins personnels. Adam Smith n'aurait pas présenté des arguments différents. Le surintendant ne craignait même pas de dire que les riches allaient économiser en dons charitables ou en larcins plus qu'il ne leur en coûterait en taxes. Plus tard, il admettait même que la discipline imposée à une population grâce à l'école permettrait d'économiser en frais de coercition! On disait franchement à l'époque des vérités qui ne sont pas moins exactes aujourd'hui. Rappelons-nous que cette population avait connu deux rébellions armées déjà, que les charivaris étaient monnaie courante; aussi des arguments au sujet de l'apprentissage de la démocratie, de la protection de la paix sociale et de la satisfaction des besoins personnels devaient trouver des oreilles attentives.

Les autorités invoquaient de façon explicite les intérêts du capitalisme et du parlementarisme pour faire la publicité des institutions scolaires. L'inspecteur Bardy que je citais plus haut allait jusqu'à affirmer: «En effet, où l'instruction élémentaire n'existe pas, le commerce languit, l'industrie agricole demeure stationnaire, l'ignorance secoue sa tête hideuse, la superstition marche vers l'incrédulité, et l'incrédulité vers la démoralisation. Sans moralité publique, que deviennent l'institution du jury, le droit d'élire? $\gg^{20}$ Le même homme, sans doute mû par le désir d'impressionner le nouveau surintendant et de garantir son emploi, déclarait ailleurs dans son rapport:

L'instruction du peuple et le crédit de l'état se tiennent donc étroitement unis; leur union seule peut produire le bien-être général qu'on pourrait définir ainsi: l'intelligence dans le travail, la rapidité dans la circulation des capitaux, le parfait entretien de toutes les voies de communication; l'ordre, la prévoyance et l'économie dans l'administration du pays; enfin, la plus exacte et la plus large répartition de la richesse publique. On a reconnu que tout large développement du bien-être matériel ne peut s'obtenir que par l'agriculture. Or, comment établir l'équilibre entre l'industrie et l'agriculture, source intarissable de produits, premier fondement de la richesse publique, si, dans chaque localité, au milieu des classes laborieuses, il ne s'élève un homme qui leur enseigne à

20 Pierre-Joseph-Olivier Chauveau, «Rapport du surintendant de l'Instruction publique pour le Bas-Canada pour l'année 1855», dans Journaux de l'Assemblée, 1856. Rapport de l'inspecteur Bardy. 
exprimer la pensée au moyen des lettres, les nombres, au moyen des chiffres; qui ouvre l'esprit du cultivateur à l'observation, au raisonnement; l'habitue à tenir un compte journalier des dépenses, des recettes, des ventes, des achats; à comparer l'état des frais à celui des produits, pour qu'il reconnaisse les procédés préférables pour chaque qualité du sol qu'il doit exploiter, qu'il sache quelles sont les opérations plus ou moins productives, les économies et les dépenses profitables à son exploitation.

A une époque, M. le surintendant, où l'industrie subit une grande transformation, où la précision et l'économie des machines tendent de toutes parts à se substituer à la force et à l'intelligence individuelles, où il est urgent d'inculquer aux classes ouvrières la nécessité d'une nouvelle instruction, sans laquelle, à chaque perfectionnement de leur art ou métier, elles seraient exposées à manquer de travail; à cette époque, dis-je, qui leur donnera spécialement les notions usuelles de mécanique, de physique, de chimie, dont tout progrès tend à rendre l'usage plus indispensable? Qui les préparera à l'exercice des diverses professions? Qui les initiera à ces principes d'hygiène, à ces notions physiologiques propres à réformer une foule d'erreurs traditionnelles, de préjugés et d'habitudes funestes? N'est-ce pas l'instituteur? ${ }^{21}$

Les élus, à la fin des années 1840 , obtinrent enfin la responsabilité ministérielle du parlement britannique... en même temps que celui-ci mettait fin à la protection douanière dont jouissaient les produits des colonies sur les marchés de la métropole. Le gouvernement du CanadaUni devait donc s'occuper de réorganiser l'économie de la colonie et négocier avec les États-Unis un traité de réciprocité. Alors les députés et le surintendant misèrent naturellement sur l'école pour favoriser la prospérité. Partout on se plaignait du grand nombre de collèges classiques qui préparaient aux professions libérales déjà encombrées, et on prônait un enseignement plus en accord avec les exigences de l'économie:

Sans les écoles modèles et académiques, il est bien à craindre que grande partie des dépenses que l'on fait aujourd'hui auxquelles le gouvernement et les habitants contribuent, pour répandre l'éducation dans le pays, soit sans bon résultat général.

C'est l'éducation pratique qu'il faut à nos jeunes gens, parce qu'elle est celle des affaires, et qu'à moins de les rendre habiles à prendre sciemment part dans toutes les transactions de la vie active, dans tous les genres d'industrie honnête, le but qu'on se propose au moyen de notre loi des écoles primaires ne serait que faiblement atteint. Dans ce cas nos jeunes gens qui, certes, ne manquent pas d'intelligence naturelle, seraient, nonobstant, en grande partie exposés à être humiliés par leur infériorité, sous le rapport de l'instruction, à devenir des prolétaires exclus des premiers emplois, 
sans pouvoir participer avantageusement aux meilleures sources de gain, ni contribuer honorablement à leur forme de gouvernement. $^{22}$

Trouverait-on une analyse différente chez les sociologues libéraux d'aujourd'hui? L'éducation avait été affaire d'État sous le Parti patriote. Elle devait l'être encore sous le Parti réformiste. Le parlementarisme, la responsabilité ministérielle augmentaient l'intérêt pour la chose publique. Il fallait que la population soit à même de participer à la vie politique, mais aussi qu'elle puisse conduire la contrée sur le chemin de la prospérité. L'implication du peuple dans la vie publique devait exister tant au niveau local qu'au niveau de l'État colonial. Voyons maintenant quelle forme allait prendre ces principes lors de la création du réseau scolaire.

\section{LA MISE EN PLACE DU RÉSEAU D'ENSEIGNEMENT PUBLIC}

Un réseau scolaire allait donc naître et se développer, et un département du gouvernement en assumer une direction de plus en plus ferme afin qu'il servît bien les objectifs décrits ci-dessus. Dans les études faites en complément de la mission d'enquête de Durham, on trouvait deux constatations lourdes de conséquences pour le réseau scolaire. D'abord, on reprochait aux écoles de syndics, dont l'administration avait été confiée à un comité permanent de la Chambre, d'avoir été un outil politique entre les mains du Parti patriote. Ensuite, on se plaignait de l'absence d'institutions municipales, qui rendait difficile la bonne administration de la colonie. Au moment où Sydenham vint occuper le poste de gouverneur, avec la mission de régler le «problème national» canadien, il voulut le faire en s'appuyant sur un nouveau réseau scolaire et sur les municipalités. Avant même que la première session de l'assemblée du Canada-Uni ne fût commencée, une ordonnance des comités spéciaux allait donner un pouvoir discrétionnaire au gouverneur sur les municipalités qu'elle créait ${ }^{23}$. Puis une loi scolaire était votée le 18 septembre 1841 , peu après que les députés se fussent réunis ${ }^{24}$.

Cette nouvelle législation était intimement liée à l'ordonnance des municipalités: les conseils de district qu'elle avait institués pouvaient

22 Jean-Baptiste Meilleur, «Rapport sur l'instruction publique dans le Bas-Canada pour l'année 1853», dans Journaux de l'Assemblée, 1853.

234 Victoria, chapitre 4 . L'institution municipale se trouvait ainsi, dans les circonstances, détournée de son objectif premier qui devait être de permettre à la population locale de participer à l'administration de la chose publique. Cela tenait, bien sûr, à la méfiance des autorités au lendemain des événements de 1837-1838. La loi des municipalités qui sera édictée en 1845 corrigera cette situation.

${ }_{24}$ 4-5 Victoria, chapitre 18. 
diviser les paroisses ou les «townships» en arrondissements d'école, distribuer à chacun de ceux-ci sa part de subvention, faire prélever les sommes nécessaires à la construction et à l'entretien des établissements d'enseignement. Chaque canton ou paroisse se devait d'élire cinq ou sept commissaires chargés de choisir et d'acheter un terrain pour l'école, de surveiller la construction de celle-ci et de l'entretenir, d'embaucher les instituteurs, d'établir les cours d'études et de choisir les manuels. Dans les cités et villes qui ne faisaient pas partie des districts (essentiellement Québec et Montréal), le conseil municipal se trouvait investi des mêmes pouvoirs au sujet des écoles. Quant au financement, les autorités locales devaient prélever une somme au moins égale à celle reçue en subvention du gouvernement. Pour ce faire, elles établiraient des taxes sur la propriété ${ }^{25}$. Avec cette loi, le législateur n'avait pas cherché à être original: il avait plus ou moins plagié une loi de l'État de New York. Il laissait aux autorités locales une large direction des institutions, plus que sous le régime des écoles de syndics. Quand on sait tous les pouvoirs que laissait au gouverneur l'ordonnance des municipalités, l'autorité des commissaires restait néanmoins toute relative. Le lien entre cette ordonnance et la loi scolaire s'avéra néfaste. Les autorités municipales n'avaient pas nécessairement envie de travailler à l'établissement d'un réseau scolaire ou n'en avaient pas la compétence.

Cette loi créait le poste de surintendant de l'instruction publique. $\mathrm{Au}$ début, on prévoyait que ce fonctionnaire serait nommé pour le Canada-Uni tout entier, avec des assistants pour chacune des deux parties du pays. Mais bientôt on trouva deux surintendants qui administraient chacun son système scolaire de façon autonome: l'objectif d'une assimilation des francophones allait d'ailleurs s'estomper rapidement. Pour le Bas-Canada, Jean-Baptiste Meilleur vint offrir ses services pour ce nouveau poste ${ }^{26}$. L'existence même de ce fonctionnaire, chargé de distribuer entre les arrondissements scolaires, au prorata des populations d'enfants s'y trouvant, l'octroi gouvernemental aux écoles, ouvrait la porte à une intervention gouvernementale sans cesse grandissante. Meilleur réclama tout de suite que les commissaires élus assument seuls la direction des écoles, et qu'ils prélèvent eux-mêmes, de façon coercitive, une taxe scolaire sur la valeur des propriétés ${ }^{27}$. Nous avons vu

25 Guy Houle, op. cit., 43-44.

Il s'agissait d'un médecin, ancien député patriote et membre du Comité permanent de la Chambre pour l'administration des écoles de syndics. Il avait aussi publié plusieurs articles sur les questions éducatives dans les journaux, rédigé des manuels scolaires et participé, avec des membres du clergé, à la création du Collège de l'Assomption.

27 Jean-Baptiste Meilleur, «Rapport du surintendant de l'Education pour le Bas-Canada, 1842-1843", dans Journaux de l'Assemblée, 1843. Le surintendant s'était vu conseiller divers modes de taxation: il penchera pour une taxe sur la valeur des propriétés. Notons au passage qu'à Montréal et à Québec, les municipalités accorderont des subventions aux commissions scolaires, qui n'auront pas à prélever elles-mêmes les taxes. 
l'importance qu'il accordait au financement populaire des écoles: cela porterait chacun à attribuer plus de valeur à l'éducation. Et puisque chacun allait tirer profit de l'instruction largement répandue, chacun devait y contribuer selon ses moyens. L'idée d'une taxation coercitive n'allait pas susciter l'enthousiasme chez la population: aussi croyait-il que si les élus locaux prélevaient et dépensaient ces sommes, cette mesure serait mieux acceptée.

La possibilité offerte aux populations locales de s'impliquer dans la chose publique, d'élire des représentants qui pourraient jouir d'une large autonomie, prélever et dépenser des taxes, embaucher les maîtres et arrêter un programme d'enseignement, témoignait fort bien du libéralisme ambiant ${ }^{28}$. Nous avons vu qu'en Grande-Bretagne, mais aussi ailleurs, comme aux Etats-Unis, l'idée d'un Etat central discret cohabitait toujours avec le rêve d'une participation active des populations locales à des institutions démocratiques ayant de larges attributions. Le Bas-Canada allait dans cette même direction. Et il ne faut surtout pas se laisser berner par les appellations de parti, s'imaginer que seuls les «rouges» étaient libéraux, et que les autres ne l'étaient pas. Ce ne sont là, largement, que des questions de nuances. Le parti de La Fontaine et de Morin avait mis sur pied un comité d'étude sur l'agriculture, il avait mis fin au régime seigneurial, il avait négocié le traité de réciprocité... et il avait accueilli dans ses rangs Chauveau qui, dès 1844, résumait son programme politique en une phrase «Le plus grand bien pour le plus grand nombre».

Je sais bien qu'au Bas-Canada, notamment dans le domaine scolaire, la tendance à la centralisation des pouvoirs peut faire croire que l'on trahissait la foi libérale en faisant trop de place à l'État. Pensons que pour mieux articuler son réseau scolaire le Haut-Canada se dota de surintendants de comté assistés d'un conseil élu, tandis que le BasCanada embaucha plutôt de nouveaux fonctionnaires, les inspecteurs, directement soumis au surintendant. Meilleur et Chauveau ont toujours prétendu que les Haut-Canadiens étaient familiers avec les institutions locales: il était donc facile là-bas de s'appuyer sur elles pour bien faire fonctionner le système scolaire ${ }^{29}$. L'effort centralisateur du Bas-Canada pouvait cependant obéir à une préoccupation libérale: pour faire passer chez la population ces valeurs libérales, peut-être fallait-il écorcher un

28 Quelques années plus tard, Meilleur se livrait à un autre «acte de foi» libéral: «Il n'y a pas dans la loi des écoles une disposition qui se rattache plus aux droits constitutionnels d'un peuple libre, à sa franchise élective, et qui doive être plus chère aux pères de famille, et dont ils devraient être plus jaloux et faire usage avec sagesse, avec honnêteté et avec conscience, que celle en vertu de laquelle ils sont par la loi appelés à faire le choix de personnes convenables pour être Commissaires d'école.» Jean-Baptiste Meilleur, «Rapport du surintendant de l'Education pour le Bas-Canada pour l'année 1846», dans Journaux de l'Assemblée, 1846.

29 Rappelons que le surintendant ontarien, Egerton Ryerson, pouvait imposer ses vues, tant en ce qui concerne l'uniformisation des programmes que dans ses efforts pour ruiner les écoles séparées. La décentralisation ontarienne pouvait n'être pas réfractaire à un pouvoir central fort. 
peu le libéralisme. Il ressort des discours ou de la correspondance des surintendants qu'ils n'étaient pas certains que laissés à eux-mêmes les commissaires n'auraient pas été victimes d'agitateurs (les éteignoirs) ou de leur propre ignorance. Bien plus, peut-on ignorer que si l'Église s'empressait de réclamer, au nom du droit naturel, que les pères de famille assument la direction de l'enseignement par le biais des commissions scolaires, cette affirmation s'accompagnait nécessairement du rappel du devoir surnaturel de l'Église qui devait, à cause de sa mission divine, avoir la haute main sur le réseau éducatif ${ }^{30}$. Pour les surintendants, il pouvait convenir d'assumer d'une main ferme la direction du système scolaire, parce qu'autrement celui-ci aurait pu devenir l'instrument d'une institution qui aurait poursuivi des objectifs étrangers au libéralisme. Les idéologies existent dans le concret et des contradictions apparentes peuvent témoigner plutôt du réalisme des attitudes.

C'est dans ce contexte que Meilleur voulait s'assurer de la compétence des candidats au poste de commissaire. Il souhaitait donc que les élus, avant d'entrer en fonction, fissent la preuve de qualifications littéraires ${ }^{31}$ et morales: il paraissait inconcevable de confier la direction des écoles à des gens qui n'auraient pas été d'une moralité éprouvée, mais aussi qui n'auraient su ni lire ni écrire. Il fallait donc s'assurer que les élus étaient aptes à l'engagement et à la direction de professeurs, au choix des manuels et à la création de programmes de formation. En même temps, les commissaires devaient prouver qu'ils étaient au-dessus de tout préjugé sur l'origine nationale, les appartenances religieuses ou les affiliations à un parti politique... Bien sûr, on ne se trouvait qu'à

30 Les auteurs qui veulent prétendre que l'Église, puisqu'elle représentait $80 \%$ des Québécois, était tout à fait démocratique lorsqu'elle réclamait un système scolaire confessionnel, oublient volontiers que celle-ci n'a jamais caché qu'elle prétendait avoir le premier rôle à jouer dans ce domaine, au nom de sa mission divine. En même temps que le droit des personnes à donner à leur progéniture une formation correspondant à leurs valeurs, elle affirmait aussi son droit à évaluer la validité des choix des parents catholiques, à orienter d'autorité ces choix puisqu'elle se disait l'interprète unique des volontés divines (ayant toujours refusé, pour ses fidèles, le libre examen des écritures). Voir à ce sujet F. Laurent, La réaction religieuse (Paris, A. Lacroix, Verboeckhoven et Cie, éditeurs, 1869), surtout le chapitre intitulé «L'ultramontanisme et la civilisation moderne», la section intitulée «Sur le droit divin d'enseigner», ou mieux encore un autochtone qui a fait autorité, Mgr Louis-Adolphe Paquet, Droit public de l'Église. L'Église et l'éducation à la lumière de l' histoire et des principes chrétiens (Québec, Imprimerie Laflamme, 2e édition, 1916), 182-183. Paquet écrivait: «De même qu'il n'est pas loisible à personne de professer la religion qui lui plaît ou de n'en professer aucune, de vivre sans foi, sans honnêteté, sans morale, on ne saurait non plus reconnaître aux parents la liberté juridique d'élever leurs enfants en dehors de la loi de Dieu, en dehors de la religion véritable, de ses principes et de ses préceptes. La religion est nécessaire à l'homme, nécessaire à l'adolescent, nécessaire à l'enfant. Et elle s'incarne dans l'Eglise catholique, unique hér.tière de l'esprit du Christ et dépositaire souveraine de ses doctrines comme de ses pouvoirs.» Ces principes n'ont rien de démocratique, rien de libéral, et ce seront ceux qui guideront les dirigeants réels du réseau scolaire francophone après 1875 . Ces dirigeants réels, ce seront les membres de l'épiscopat, au sein du comité catholique du CIP.

Jean-Baptiste Meilleur, «Rapport du surintendant de l'Education pour le Bas-Canada pour l'année 1846», dans Journaux de l'Assemblée, 1846. 
quelques années de l'époque où ces questions avaient conduit à la guerre civile! Mais cela rejoignait aussi l'idée libérale de l'égalité devant la loi... et devant l'école. Le surintendant désirait de plus des commissions scolaires indépendantes des municipalités, précisait les compétences à exiger des commissaires et suggérait un mode de taxation coercitif. Il allait aussi jusqu'à revendiquer l'enseignement obligatoire:

L'obligation imposée aux parents d'envoyer leurs enfants à l'école, sous peine d'amende, est une mesure de rigueur qui forcerait les parents à procurer à tous leurs enfants indistinctement le bienfait de l'éducation, et s'ils ne savent pas encore quel est le plus grand bien que l'on puisse faire à leurs enfants, il est temps de leur apprendre à le connaître par l'importance et par la sévérité que l'on mettra dans ces mesures obligatoires à cet effet.

(...) ... cette obligation ne comprendrait que les enfants âgés de sept à douze ans inclusivement, pendant six mois de l'année seulement. $^{32}$

Enfin, on verra plus loin qu'un bureau d'examinateur allait encore élargir les prérogatives du Département.

Déjà, on voyait se dessiner la tendance de l'État à façonner un système scolaire susceptible de rencontrer les idéaux politiques précédemment énumérés. Mais la loi de 1841 ouvrait aussi la porte à une autre tendance. La première version ne faisait aucune allusion aux divergences religieuses existant dans la population et prévoyait plutôt un enseignement moral qui ne heurterait les membres d'aucune confession. Le clergé catholique, comme celui d'autres sectes, ne tarda pas cependant à contester cet aspect du projet et un amendement à l'article XI reconnut le droit de dissidence. Le législateur permettait en effet que dans une municipalité la minorité religieuse ait le droit de déclarer dissidence et d'établir sa propre école confessionnelle. La loi ne reconnaissait pas de caractère confessionnel aux écoles de la majorité ni aux écoles des arrondissements où il n'y avait pas eu dissidence, mais nombre d'auteurs prétendent qu'il y a eu, dès lors, confessionnalité de fait ${ }^{33}$. Dans une localité où la population était entièrement de la même allégeance religieuse, nous pouvons admettre en effet que le pasteur et les

32 Ibid

33 Jean-Yves Lord, L'aspect législatif de la confessionnalité scolaire aux 19e et 20e siècles, thèse de maîtrise (théologie), Université Laval, 1974. Cet auteur renvoie à Groulx, Carignan, Audet et Bilodeau, tous de son avis. Répétons que Houle, op. cit., affirme qu'au Québec seules les commissions scolaires de Montréal et Québec, et les écoles dissidentes, ont un caractère confessionnel selon la loi, et seules elles ont leur confessionnalité «constitutionnellement» protégée. En 1871 le recensement nous apprend que Montréal compte 107225 habitants et Québec 59699 , et 1191516 pour la province. En 1864, sur 3048 écoles au Bas-Canada, on trouve 182 écoles dissidentes (dont 134 protestantes). Et rappelons que les écoles protestantes, tout à fait chrétiennes bien sûr, sont en réalité «multi-confessionnelles». En Ontario Ryerson aurait dit «communes»... Je laisse le lecteur mesurer combien peu d'écoles étaient confessionnelles catholiques. 
commissaires pouvaient éventuellement infléchir les contenus d'enseignement et les pratiques pédagogiques et créer une école confessionnelle de fait. Mais, dans le cas des localités où se côtoyaient des habitants de diverses sectes (et on ne voyait pas apparaître une école dissidente dans chacune d'elles, loin de là!), les écoles ont dû demeurer «communes».

En réaction aux propositions d'amendements faites par Meilleur, la Chambre allait promulguer les lois de 1845 et 1846, à propos desquelles on a parlé de «Grande Charte» scolaire du Québec, qui garantissait les droits de la famille et des autorités locales. La Fontaine avait voulu, dès 1843 , présenter une nouvelle loi, mais il fallut attendre 1845 avant qu'elle ne voit le jour ${ }^{34}$. Bien qu'elle ait élargi, comme on le verra, le contrôle du surintendant sur les commissions scolaires, des auteurs attachés à la vision cléricale traditionnelle de l'école bénirent cette législation qui accordait, selon eux, une place prépondérante à la famille dans les questions scolaires ${ }^{35}$. Pour Meilleur, elle faisait figure de catastrophe et son rapport pour cette année-là ne fut en conséquence qu'une longue succession de griefs. Il indiquait, dès le début, pour donner le ton de tout son texte:

En voulant rendre compte des causes principales de ce malheur [le non-fonctionnement de la loi], je sens que j'ai à remplir une tâche plus pénible encore que difficile: mais je ne dois pas, par une faiblesse déplacée, hésiter à les [défauts de la loi] signaler à l'autorité compétente, afin de la mettre à même de prescrire un remède proportionné au mal. ${ }^{36}$

Pour lui, deux raisons entraînaient des difficultés à faire exécuter la loi. D'abord elle était mal rédigée! Il disait à ce sujet:

Passés à la hâte en comité général de toute la Chambre, à la fin de la session dernière, ensemble avec l'Acte des municipalités rurales, et grossoyés tous deux dans la même nuit, veille du jour où le Parlement fut prorogé, il était impossible que ces deux Actes qui, d'ailleurs, demandent plus d'expérience que nous n'en avons encore pu obtenir pour en faire des Actes parfaits, eussent pu être sans défaut. ${ }^{37}$

348 Victoria, chapitre 41 . Le retard tient à une prorogation surprise de la session et aux difficultés liées à la lutte pour la responsabilité ministérielle. Voir J. Monet, op. cit.

${ }_{35}$ Groulx et d'autres historiens cléricaux, dont Lionel Allard et Gérard Filteau, Un siècle au service de l'éducation 1851-1951. L'inspectorat des écoles dans la province de Québec (Québec, Ministère des Communications, Service des impressions de régie, s. d.), 2 tomes, essayaient d'étayer sous Duplessis la résistance à tous les changements en cherchant les racines historiques les plus lointaines à ce qu'ils appelaient «le meilleur système scolaire du monde». Même L.-P. Audet était très réservé sur le rôle du clergé en éducation. Voir la note 30 à ce sujet.

36 Jean-Baptiste Meilleur, «Rapport du surintendant de l'Education pour le Bas-Canada

pour l'année $1846 »$, dans Journaux de l'Assemblée, 1846 .
37 Ibid. Meilleur exprime bien ici son impression que nous étions dans l'«enfance des institutions». 
Puis la liaison, encore une fois, d'une loi des écoles avec une loi des municipalités exigeait une collaboration de ces dernières, ce qui ne s'obtenait pas toujours. Le texte de la loi était si ambigu que les autorités municipales et les commissaires n'osaient passer à l'action, pour le prélèvement des cotisations ou l'utilisation de l'octroi gouvernemental notamment, de crainte d'empiéter sur les prérogatives de l'autre institution $^{38}$ !

La liste des amendements que suggérait Meilleur était fort longue, mais surtout il s'opposait d'abord à ce que la loi, au mépris de ses recommandations, ait prévu une contribution scolaire volontaire plutôt qu'une taxe coercitive. Parmi les amendements proposés, certains revêtaient un caractère urgent ${ }^{39}$. De ces suggestions résulta la loi de $1846^{40}$. L'organisation scolaire devait encore doubler l'organisation des paroisses et des cantons, mais les commissions scolaires devenaient indépendantes des autorités municipales, placées sous le contrôle des commissaires mandataires du peuple et d'un surintendant représentant la Couronne. La loi fixait précisément les conditions d'élection et prévoyait un mécanisme de contestation de celle-ci. A la satisfaction de Meilleur, les commissaires perdaient un peu de leur autonomie: ils ne pouvaient plus renvoyer les instituteurs que pour cause d'incapacité, de négligence à remplir leurs devoirs, d'insubordination, d'inconduite ou d'immoralité, après mûres délibérations, lors d'une assemblée convoquée à cette fin; dès 1852 plutôt qu'en 1856, comme l'avait prévu la loi précédente, ils devraient n'embaucher que les instituteurs possédant un certificat d'un bureau d'examinateurs (nous reviendrons sur ceux-ci); la population pouvait contester une décision de la Commission, et, dans ce cas, le surintendant rendait une sentence sans appel; etc. Cependant, Meilleur n'avait pas tout obtenu, et il regrettait qu'on n'exigeât pas une instruction minimale de ceux qui se présentaient comme commissaire. On leur demandait plutôt d'être propriétaire. Cela entraînait des choix malheureux de candidats qui, soit ne comprenaient rien à la tâche qu'ils devaient effectuer, soit étaient résolus à saboter l'effet de la loi des écoles, ou encore les deux. Les gens instruits étaient plutôt rares et on ne trouvait pas plus d'hommes riches: «Je connais même nombre de localités, d'établissements nouveaux surtout, écrivait-il, où il est impossible de trouver un nombre suffisant d'hommes qualifiés au terme de la loi actuelle pour être Commissaire d'École. Quelquefois même, il ne s'en trouve pas un seul.» ${ }^{41}$

\footnotetext{
38 Ibid.

39 Ibid. Nommément, 1: rendre l'acte permanent; 2: l'émanciper de l'acte des municipalités.

409 Victoria, chapitre 27.

41 Jean-Baptiste Meilleur, «Rapport du surintendant de l'Education pour le Bas-Canada pour l'année 1846», dans Journaux de l'Assemblée, 1846.
} 
Dès 1841 , la loi prévoyait la création de Bureau d'examinateurs, dont les membres devaient être nommés par le gouverneur-en-conseil. Ils allaient décerner des certificats de compétence aux instituteurs (sauf dans le cas de religieux, de prêtres ou de pasteurs), et bientôt aux institutrices. Puisque ces certificats allaient devoir préciser le niveau de qualification des candidats à l'enseignement aux écoles élémentaires, modèles ou académiques, indirectement le Bureau établissait au moins brièvement les programmes de chacun de ces niveaux d'enseignement:

Les connaissances requises des candidats à l'enseignement seraient pour un Instituteur d'Académie, celles de toutes les branches d'une éducation classique, parce que ces Instituteurs sont destinés à y préparer les élèves; pour les Instituteurs d'Ecoles-Modèles, celles qui les rendraient capables d'enseigner avec succès la lecture, la grammaire correctement dans sa langue, ou de celle de la majorité des enfants allant à l'école, l'analyse des parties du discours, à écrire et l'arithmétique dans toutes ses parties, la tenue de livres, le dessin linéaire, les éléments de mesurage, la sphère et la géographie, et commencer par celle de l'Amérique du Nord; et pour les Instituteurs des Ecoles Elémentaires ils devraient être habiles à enseigner avec succès la lecture, l'écriture, les éléments de la grammaire et l'arithmétique au moins jusqu'à la règle de trois inclusivement. ${ }^{42}$

Le même organisme devait aussi s'occuper du choix des manuels. A ce sujet, Meilleur voulait d'abord éviter l'utilisation de livres américains, imbus de principes républicains, mais aussi favoriser la publication de manuels canadiens (ou britanniques, à la rigueur) uniformes pour chaque matière:

Les Commissaires d'Education, étant libres de choisir parmi les livres que la loi laisserait à leur disposition ceux qui leur conviendraient le mieux, feraient probablement tomber leur choix sur une seule espèce pour chaque sujet, s'ils prenaient là-dessus l'avis du Surintendant qui, dans tous les cas, n'aurait toujours qu'une voix consultative et rien de plus. ${ }^{43}$

42 Jean-Baptiste Meilleur, «Rapport du surintendant de l'Education pour le Bas-Canada pour l'année 1846", dans Journaux de l'Assemblée, 1846. Les bureaux d'examinateurs ne seront établis qu'après les législations de 1845-1846, dans les villes de Québec et Montréal, quoiqu'ils aient été prévus dans la loi de 1841 . Meilleur donne ici une bonne idée des programmes de chaque niveau de formation, bien que la loi laissait aux commissaires la responsabilité d'établir les programmes.

43 Ibid. Le surintendant disait encore en 1846: «Les Bureaux d'examinateurs ont en outre le pouvoir de prescrire les livres qui doivent être en usage dans les écoles qui sont sous le contrôle de la loi, et les Commissaires d'Ecole tenus aux deux espèces d'instituteurs désignés dans la 50e clause, et ne pouvant légalement permettre, dans les écoles sous leur juridiction, l'usage de livres non approuvés par ces bureaux, il s'ensuit que les cours d'étude à être suivis dans chaque école élémentaire ou modèle, ainsi que l'espèce de livres qui doivent y être en usage, sont pourvus et réglés par la loi. Exceptés cependant les livres qui ont trait à la morale et à la religion, dont le choix est du ressort exclusif du prêtre ou du ministre desservant pour l'usage des enfants des écoles de sa croyance religieuse.» 
Il restait bien peu de choses aux commissaires, dont la loi disait pourtant qu'ils devaient embaucher les maîtres, administrer les écoles et choisir les programmes...

Pourtant, existaient dans la société bas-canadienne des éléments qui auraient voulu augmenter encore le rôle de l'État, soit en donnant un pouvoir plus direct à la Chambre et aux députés (on reconnaît là une certaine nostalgie du comité permanent d'éducation, au temps du régime des écoles de syndics), soit en augmentant tellement les pouvoirs du surintendant qu'il aurait pu forcer seul l'application de la loi scolaire sur tout le territoire. Meilleur opposait à ces suggestions des libéraux radicaux que cela aurait exposé la loi scolaire à l'opposition d'une partie de la population (celle qui n'aurait pas voté en faveur du parti majoritaire), que cela l'aurait rendue odieuse à tous les clergés et aussi au peuple à cause de l'absence de toute participation au niveau local. Il préférait un partage des responsabilités entre des commissaires élus et un surintendant au service du gouvernement, mais non-impliqué dans l'un ou l'autre des partis. Ces discussions sur la nature du système scolaire public étaient d'autant plus légitimes que la taxation coercitive introduite par la loi de 1846 faisait naître des mouvements d'opposition importants, surtout dans la région du lac Saint-Pierre ${ }^{44}$. Ces contestataires étaient les «éteignoirs» ${ }^{45}$ et leurs actions suscitèrent sans doute les derniers grands charivaris liés à des questions politiques.

Dans ce contexte d'opposition, le clergé catholique exprimait aussi, notamment par la bouche de Mgr Bourget, le désir d'accaparer le réseau scolaire. Meilleur devait refuser tout doucement cette suggestion pour ne pas se mettre à dos cette institution de plus en plus puissante dans la société bas-canadienne, en plaidant d'abord que ce serait imposer à l'Église une tâche qui la forcerait à négliger son «saint ministère», qui la rendrait odieuse à une partie de la population en l'obligeant à se mêler aux querelles politiques, la ferait se heurter à l'opposition de personnes malveillantes. En fait, le surintendant savait bien que dans une colonie où cohabitaient les membres de diverses confessions religieuses, il aurait été impossible de donner à l'un des clergés un rôle majeur dans l'organisation scolaire sans susciter maints problèmes, sans compter que cela aurait contredit les présupposés idéologiques décrits plus tôt. Aussi croyait-il que les clergés ne devaient avoir que le contrôle de l'éducation morale et religieuse, à moins que la volonté des contribuables, librement exprimée par voie d'élection, n'appelât certains de leurs membres à prendre plus directement part à la régie locale des écoles: ils pouvaient en effet être élus commissaires. Mais une fois élus, ils

\footnotetext{
44 Edouard Fournier, L'abbé, La guerre des éteignoirs, mémoire de licence ès lettres, Université Laval, 1954. On pourra consulter aussi les ouvrages de L.-P. Audet à ce sujet.

45 Les contemporains «éclairés» utilisaient ce terme pour désigner ceux qui risquaient d'«éteindre» la lumière de la connaissance.
} 
avaient le loisir de se retirer si le commerce des autres commissaires leur répugnait, alors qu'un commissaire laïc ne pouvait, à toutes fins utiles, démissionner de ses fonctions.

Meilleur repoussait aussi l'idée de la présence de deux surintendants, l'un pour les catholiques, l'autre pour les protestants, que certains mettaient de l'avant:

Le principe d'un semblable système, s'il était admis, ferait peu d'honneur au pays au point de vue de la civilisation et de la libéralité de ses habitants, et ce, d'autant moins qu'il pourrait être réclamé à l'infini; car il est parmi les protestants nombre de sectes religieuses, différentes et exclusives, qui sont aussi opposées les unes aux autres par la foi, qu'elles le sont toutes à l'église catholique romaine. ${ }^{46}$

Le surintendant semblait même enclin à croire que le régime des écoles communes valait mieux que l'introduction du droit à la dissidence ${ }^{47}$. Visiblement, il essayait de louvoyer entre des libéraux plus audacieux que son gouvernement et une Église qui augmentait ses forces dans le but avoué d'exercer un jour le leadership au sein de la communauté francophone. Et il arrivait, je crois, à préserver le caractère libéral du réseau scolaire, quoique les concessions qu'il devait faire laissaient présager de la grande catastrophe de $1875^{48}$. Il dut même concéder aux ministres du culte, en récompense pour leurs bons services lors de la guerre des «éteignoirs», le droit d'être élus commissaires même s'ils ne possédaient pas la qualification foncière requise $e^{49}$.

Meilleur était largement satisfait de la loi de 1846 et il ne recommandait pas d'amendements. Au contraire, il voulait que la loi reste inchangée de peur qu'en cas de modifications le peuple pût croire que les désordres pouvaient faire reculer les législateurs: cela aurait été, croyait-il, ouvrir la porte à l'anarchie. Tout au plus réclamait-il ces inspecteurs qui allaient en permettre une meilleure application. La loi de 1849 permettait au surintendant de faire nommer des «députés» qu'il pouvait envoyer sur le terrain faire enquête et proposer des solutions aux différents problèmes, puisque ses autres tâches l'empêchaient de

\footnotetext{
46 Jean-Baptiste Meilleur, «Rapport du surintendant de l'Education pour le Bas-Canada pour l'année 1846", dans Journaux de l'Assemblée, 1846. Ici, Meilleur réfère aux idéaux d'une population libérale. Si Montréal et Québec faisaient exception, leurs commissions scolaires ne pouvaient refuser un enfant sous prétexte de sa foi, elles demeuraient «libérales» au moins dans le recrutement. Ce n'est que plus tard qu'on recrutera les enfants exclusivement dans l'un ou l'autre des deux groupes religieux.

47 J'insistais plus haut sur le faible nombre d'écoles dissidentes à la veille de 1867

48 Les Québécois, me semble-t-il, ont payé cher l'abandon du système scolaire à l'épiscopat, notamment parce qu'ils n'ont jamais pu, avant 1960, intégrer complètement les politiques scolaires à une politique globale de développement, comme je l'ai démontré ailleurs. Jean-Pierre Charland, Histoire de l'enseignement technique et professionnel (Québec, Institut québécois de recherche sur la culture, 1982), 482.

$49 \quad 12$ Victoria, chapitre 50
} 
se rendre lui-même dans les écoles. A la faveur de la guerre des «éteignoirs», Meilleur s'en remit à l'Exécutif en précisant que les oppositions risquaient d'abattre complètement le système scolaire. Il exigeait des assistants afin d'être en mesure d'assurer l'application de la loi. Le gouvernement se rendit finalement à ses demandes. Augustin-Norbert Morin prépara un projet de loi qu'il présenta d'urgence pendant les derniers jours de la session de $1851^{50}$. Adoptée la veille de la prorogation des Chambres, elle créait le service d'inspectorat. Elle permettait au gouverneur de nommer de temps à autre des inspecteurs d'écoles élémentaires dans le Bas-Canada. Ils devaient agir en vertu des directives du surintendant et lui faire rapport tous les trois mois pour décrire l'état de l'enseignement. Notons au passage que tout inspecteur nommé en vertu de cette loi se trouvait «ex officio» juge de paix de son district.

Les premiers inspecteurs furent nommés le 2 mars 1852, au nombre de 23, pour autant de districts. Beaucoup n'avaient pas de compétence particulière en ce qui concerne l'éducation et l'un d'eux admettait candidement:

\begin{abstract}
MONSIEUR, Immédiatement après la réception de la lettre du secrétaire provincial en date du 20 septembre 1853, et aussi de la commission me nommant inspecteur des écoles protestantes de la cité de Québec, je me mis en mesure de m'initier aux devoirs de cette charge qui venait de m'être conférée, par une étude des actes du parlement relatifs à l'éducation en cette province, par des entrevues avec vous, auxquelles vous avez accédé avec tant de bienveillance, et finalement par l'examen attentif et consciencieux de votre code précieux d'instructions imprimées et manuscrites. ${ }^{51}$
\end{abstract}

Ces inspecteurs devaient le plus souvent leur nomination à la lutte qu'ils avaient faite aux «éteignoirs» et ils jouissaient d'une bonne réputation dans leur milieu. C'était heureux, puisqu'on les lançait souvent dans des localités où les autorités voulaient entraver l'application de la loi des écoles. Soulignons au passage que le caractère confessionnel du réseau scolaire s'accentuait un peu: certains inspecteurs s'occupaient spécifiquement des écoles protestantes de Québec et de Montréal et des écoles dissidentes protestantes du reste de la province.

Appelés à être les yeux et les oreilles du surintendant, les inspecteurs se voyaient confier une rude besogne: visiter toutes les municipalités scolaires de leur district à tous les trois mois et examiner les maisons d'école, les opérations des commissaires, tenir des statistiques scolaires, rendre compte de la composition et de la compétence du

so 14-15 Victoria, chapitre 97.

51 Jean-Baptiste Meilleur, «Rapport du surintendant de l'Instruction publique pour le BasCanada pour l'année 1853», dans Journaux de l'Assemblée, 1854. Rapport de l'inspecteur Adamson. On trouvait chez les premiers inspecteurs 8 notaires, 5 médecins, 2 avocats, 1 arpenteur, 1 marchand et 5 instituteurs. 
personnel enseignant (et donner un certificat temporaire à ceux qui ne s'étaient pas encore présentés devant un bureau d'examinateurs), classifier les écoles, favoriser l'enseignement de certaines disciplines et l'usage de certains manuels et faire la publicité de certaines méthodes pédagogiques $^{52}$. Dans les trois années qui suivirent ces premières directives, le surintendant allait adresser à ses inspecteurs douze circulaires, dans lesquelles il abordait tous les sujets. Les effets ne furent pas longs à venir: alors que depuis plusieurs années le nombre des écoles se maintenait autour de 1800 , il passait à 2277 en $1852-1853^{53}$. Meilleur fut suffisamment enthousiaste pour remettre un rapport spécial au secrétaire provincial sur l'excellence du travail des inspecteurs.

Nous savons que les lois précédentes laissaient aux commissaires la régie interne des écoles, notamment en faisant des règlements d'école. Les inspecteurs constatant que ces règlements n'existaient généralement pas, ils durent en promulguer qui réglaient minute par minute le fonctionnement quotidien des institutions. Même les matières à enseigner et les manuels y étaient précisés. Ces initiatives inquiétèrent suffisamment Meilleur pour qu'il intervint dans un souci de préserver l'uniformité ${ }^{54}$. Avec les inspecteurs, il pouvait s'assurer que le réseau scolaire suivait la bonne direction, véhiculait les valeurs précédemment décrites. Pourtant une autre époque de centralisation restait encore à venir.

\section{LE DERNIER GRAND EFFORT DE CENTRALISATION}

Bien que plusieurs des difficultés du système scolaire s'étaient trouvées aplanies grâce à l'action des inspecteurs, ceux-ci se plaignaient dans leur rapport d'un manque de direction ferme du réseau. A la requête de Meilleur, le gouvernement nomma un comité d'enquête, avec à sa tête Louis-Victor Sicotte, député de Saint-Hyacinthe. Il allait adresser aux membres du clergé, aux secrétaires des commissions scolaires et à des citoyens éminents des questions sur le réseau scolaire ${ }^{55}$. Le comité étudia aussi les rapports des inspecteurs. Il allait tirer de cet examen des conclusions bien négatives: la formation donnée était trop superficielle, seule une minorité des institutrices détenaient un certificat et, dans bien des cas, même celles-là n'avaient aucune compétence; les écoles étaient sous-équipées (les quatre-cinquièmes n'avaient pas de cartes géographiques, les tableaux noirs manquaient souvent) et une

52 On trouve ces circulaires en annexe aux rapports du surintendant. Elles sont imprimées et adressées aux inspecteurs, aux commissaires et parfois aux instituteurs. On en retrouve une bonne description dans Gérard Filteau et Lionel Allard, op. cit.

53 Gérard Filteau et Lionel Allard, ibid., 32.

54 Ibid., 55ss, et Jean-Baptiste Meilleur, «Rapport sur l'Instruction publique dans le BasCanada pour 1854», dans Journaux de l'Assemblée, 1854-1855. «Circulaire no 10 aux inspecteurs".

55 Meilleur aurait conseillé à ces personnes de ne pas répondre à ces questions, sans doute parce qu'il se défiait des membres du comité. Il se mettait cependant dans une position intenable. 
bonne moitié des commissaires étaient analphabètes ${ }^{56}$. On peut résumer ainsi ses recommandations:

- Donner une direction plus ferme, plus énergique à l'enseignement;

- Nommer de meilleurs inspecteurs, les mieux payer;

- Réduire le nombre de commissaires et exiger qu'ils soient plus instruits;

- Créer des écoles normales;

- Mieux payer les instituteurs et institutrices;

- Obtenir des manuels scolaires uniformes et mieux faits;

- Prévoir de meilleurs budgets gouvernementaux pour l'éducation.

Ce jugement sévère à l'égard du système scolaire était en même temps lourd de reproches sur le travail de Jean-Baptiste Meilleur, quoique celui-ci ait déjà dans le passé recommandé des changements allant dans le même sens. Il demanda bientôt un nouveau poste qui lui permettrait de toucher le même salaire et glissa même un mot de l'emploi de maître de poste à Montréal... Pierre-Joseph-Olivier Chauveau lui succéda donc en 1855. Il s'agissait d'un député important dans le parti de La Fontaine (auquel George-Etienne Cartier avait déjà succédé) mais dont les idées libérales avaient pu inquiéter cette formation politique de plus en plus conservatrice qui venait de faire sa liaison avec les conservateurs haut-canadiens. Privé depuis peu de son portefeuille, cet emploi allait permettre de le dédommager. Le nouveau surintendant garda cependant d'excellentes relations avec le cabinet dont il avait fait partie, ce dont Meilleur n'avait jamais bénéficié. Sans compter qu'il était avantageusement connu comme journaliste, orateur et romancier ${ }^{57}$.

Dans son premier rapport, celui de 1855, Chauveau traçait son programme et reconnaissait ses lourdes dettes à l'égard de son prédécesseur. Son premier souci était l'étendue des pouvoirs des commis-

56 Boucher de la Bruère, Le Conseil de l'Instruction publique et le Comité catholique, 26. On pourrait croire que si Chauveau, comme Meilleur avant lui, avait été «libéral», le clergé l'aurait combattu. Mais, il fallait que celui-ci ait obtenu suffisamment d'influence avant de se lancer dans une pareille croisade: même en 1855, cela n'était pas encore évident. De plus, alors que les relations allaient s'améliorant entre l'Église et le Parti libéral-conservateur, aurait-il été prudent de contester les choix de ce dernier? Enfin, Chauveau avait tout de même eu maille à partir avec le clergé et il dut s'entendre avec lui sur la question de la direction des écoles normales francophones. Et, plus tard, il allait se heurter aux efforts de l'Église pour l'écarter du réseau scolaire (il avait désiré obtenir le poste de surintendant en 1875, promettant de «tout donner à l'Eglise», tellement ses problèmes financiers étaient graves). On peut même lire à son sujet: «Il [Chauveau] a aussi à subir un harcèlement continuel des ultramontains. En 1884 et 1885, JulesPaul Tardivel et le père Joseph Grenier mènent contre l'ex-surintendant une campagne de dénigrement parce que celui-ci avait déjà correspondu avec Victor Duruy et avait accepté les décorations d'un gouvernement français prétendu maçonnique. Ils l'accusent d'avoir tout au long de sa vie répandu «des idées funestes» et d'avoir perverti «jusqu'à la notion chrétienne d'éducation»: «il a déposé en germe dans nos lois et notre système scolaire (...) toutes les idées du programme maçonnique».» Dictionnaire biographique du Canada, XI: 203. 
saires. Il citait à ce sujet le rapport de l'inspecteur Hubert: «Ordre renversé des pouvoirs administratifs dans le département, première cause du mauvais fonctionnement du système: trop d'autorité discrétionnaire chez les commissaires d'école; pas assez de contrôle sur eux de la part du chef du département et de ses députés, les inspecteurs d'école. ${ }^{58}$ Le surintendant enchaînait en disant:

Le minimum de salaire que l'on fixerait en faveur des maîtres, l'obligation que l'on ferait aux institutrices d'obtenir des diplômes, (car c'est en grande partie la concurrence des institutrices sans diplômes, qui ruine les instituteurs et favorise l'avarice de quelques municipalités) le pouvoir que l'on conférait aux inspecteurs (sauf appel au surintendant ou au conseil de l'instruction publique) de renvoyer des maitres même munis de diplômes, qui mériteraient l'expulsion par leur négligence ou leur mauvaise conduite, enfin, le choix des livres d'école qu'on laisserait exclusivement au département; toutes ces restrictions importantes que l'on devrait mettre de suite aux pouvoirs illimités des commissaires remédieraient à bien des abus. ${ }^{59}$

Chauveau allait donc essayer de donner, par voie de règlements, une certaine sécurité d'emploi aux instituteurs et institutrices, toute limitée bien sûr. Il entendait aussi pouvoir empêcher la nomination de tout indésirable comme commissaire. Il ne croyait pas à la possibilité d'exiger des candidats une «qualification littéraire», car on n'aurait pu aller plus loin que leur demander de savoir lire et écrire. Il envisageait cependant de fixer une date après laquelle tout fonctionnaire provincial ou municipal devrait pouvoir lire et écrire de sa main le serment d'office avant de prendre sa charge. Cela n'aurait pu que stimuler le zèle de chacun à l'égard de l'éducation.

Chauveau voulait aussi mettre fin à l'excessive variété des manuels: un inspecteur avait signalé que dans une école tous avaient une grammaire différente. Elle tenait à la fois à la rareté des livres et à la pauvreté des habitants: les commissaires permettaient qu'on se serve des manuels qui tombaient sous la main. Les instituteurs eux-mêmes, qui changeaient fréquemment d'employeurs, ajoutaient bien involontairement à la confusion: d'une année à l'autre une école pouvait changer de maître et de programme. Le Haut-Canada avait adopté les programmes des écoles nationales d'Irlande ${ }^{60}$. On ne pouvait faire de même au BasCanada, à moins de les traduire en français, mais même là les différences culturelles auraient difficilement permis aux francophones de s'y retrouver. Chauveau préférait que le département d'Instruction publi-

\footnotetext{
58 Pierre-Joseph-Olivier Chauveau, «Rapport sur l'Instruction publique dans le Bas-Canada pour l'année 1855», dans Journaux de l'Assemblée, 1856.

59 Ibid.

60 Ce choix s'expliquait du fait que, en Irlande aussi, les écoles abritaient des catholiques et des protestants.
} 
que prit la responsabilité de la rédaction de manuels avec un contenu national. Ces livres pourraient même être imprimés aux frais de l'État et être distribués aux municipalités scolaires. François-Xavier Garneau allait le premier donner une histoire du Canada à cette série de manuels ${ }^{61}$. Le surintendant voulait aussi que le département ait un dépôt de cartes, de globes, de planétaires et d'autres matériaux pédagogiques où les commissaires pourraient s'approvisionner. Il croyait que toute école devait avoir, au moins, un tableau noir, une mappemonde et une carte du Canada.

L'époque était aux réformes de l'éducation, d'autant plus que le Canada-Uni jouissait d'une prospérité sans précédent. Dans son rapport Chauveau avait fait part de ses attentes. Les libéraux firent de même: ils rêvaient d'une société où la religion n'aurait été qu'affaire privée qui n'aurait pas interféré dans les affaires publiques. Toutes les écoles devaient être neutres, gratuites et financées par le gouvernement. Dans un discours à propos des nouvelles législations, le député Papin pouvait en effet déclarer:

...qu'il serait désirable d'établir dans toute la province un système général et uniforme d'éducation élémentaire gratuite et maintenue aux frais de l'Etat, et que les écoles ainsi établies, soient ouvertes indistinctement à tous les enfants en âge de les fréquenter sans qu'aucun d'eux soit exposé par la nature de l'enseignement qui y sera donné à voir ses croyances ou opinions religieuses violentées ou froissées en aucune manière. ${ }^{62}$

Bien sûr, les conservateurs étaient au pouvoir et ils étaient de plus en plus liés à l'Église. Ils ne pouvaient aller aussi loin. George-Étienne Cartier, secrétaire de la province dans le cabinet Taché-Macdonald, présenta en Chambre deux nouvelles lois concernant l'éducation. La première, intitulée «Acte pour amender les lois des écoles communes au Bas-Canada ${ }^{63}$, donnait aux commissaires et syndics le droit de fixer la rétribution mensuelle pour chaque enfant et de la percevoir. C'était cependant le seul élargissement des pouvoirs des commissaires. Le gouverneur-en-conseil se donnait le pouvoir de créer de nouveaux bureaux d'examinateurs; le surintendant, celui de décréter le prélèvement d'une taxe spéciale dans une municipalité scolaire pour le paiement de dettes reconnues ou résultant de jugements de tribunaux, si celle-ci ne pouvait s'acquitter autrement de ses obligat ons. Le surintendant surtout, avec l'approbation du gouverneur-en-conseil, pouvait refuser de payer la totalité ou toute partie de l'octroi d'une municipalité

\footnotetext{
61 Pierre-Joseph-Olivier Chauveau, «Rapport sur l'Instruction publique dans le Bas-Canada pour l'année 1855», dans Journaux de l'Assemblée, 1856. Suivaient d'autres suggestions, comme la création d'écoles normales et la publication dans les deux langues d'un journal d'éducation.

62 'Boucher de la Bruère, op. cit., 32.

6319 Victoria, chapitre 14.
} 
scolaire où ses instructions légitimes ou celles du Conseil de l'Instruction publique n'avaient pas été respectées, ou quand des instituteurs non-qualifiés avaient été engagés, ou quand un instituteur qualifié avait été démis de ses fonctions sans que soient respectés les délais prévus par la loi, etc. Donc cette loi reconnaissait clairement un réel contrôle au surintendant.

Plus encore, c'est par la clause qui prévoyait la création du Conseil de l'Instruction publique que cette loi minait les responsabilités des commissaires. Ce conseil recevait en effet le contrôle des écoles normales comme celui des bureaux d'examinateurs: la formation professionnelle des instituteurs et l'examen de leur compétence lui revenaient donc. Bien plus, le conseil, avec l'approbation du gouverneur-en-conseil, ferait les règlements qu'il jugerait à propos pour l'organisation, la gouverne, la discipline et la classification des écoles communes (cette possibilité de réglementer s'appliquait, bien sûr, aux écoles dissidentes: il n'y avait qu'un régime d'éducation au Bas-Canada) et la classification des instituteurs. Le conseil choisirait ou ferait publier, toujours avec l'approbation du gouverneur-en-conseil, les livres, les cartes, les globes dont on se servirait à l'exclusion de tous les autres dans les écoles élémentaires, modèles ou académiques sous le contrôle des commissaires ou des syndics, ceci en tenant compte de la langue d'enseignement dans les écoles; mais ce pouvoir excluait le choix des livres de religion et de morale qui revenait au ministre du culte. Enfin le conseil pouvait révoquer le certificat de qualification d'instituteurs ou d'institutrices pour manquement aux bonnes moeurs, à la bonne conduite ou à la tempérance.

Le législateur créait donc un organisme composé de personnes pesant du poids de leur «auctoritas» qui allait assumer une très large part de la direction des écoles: une mesure toute libérale, susceptible de faire mieux accepter à la population les mesures scolaires. Le CIP ne fut créé que le 17 décembre 1859 par un arrêté ministériel, alors que la loi avait été votée en 1856 . On y trouvait dix catholiques et quatre protestants, avec en plus le surintendant. Étienne-Paschal Taché, vieux politicien, ancien premier ministre-associé, chef pendant un temps de la section bas-canadienne du Parti conservateur, fut élu président du conseil par les autres membres. Il avait été proposé par l'évêque anglican de Montréal, avec l'appui de l'évêque catholique de la même ville! Le conseil établissait les règlements pour sa régie interne et formait quelques sous-comités auxquels on attribuait des tâches spécifiques: sous-comité pour établir des règlements pour le choix des manuels et du matériel pédagogique; sous-comité pour la régie du cours d'études des écoles normales; deux autres pour la classification et la discipline des écoles publiques et la formation des bureaux d'examinateurs. Dès le début, le Conseil de l'Instruction publique demanderait que la loi soit modifiée afin que les membres catholiques et les membres protestants 
puissent voter seuls sur les questions qui concernaient plus directement une confession religieuse. Ces sous-comités commençaient leurs travaux le 11 janvier $1860^{64}$.

Nous voulons ici donner le point de vue de Gérard Filteau, souvent enclin à chanter les louanges de l'administration du système scolaire par les commissions scolaires et l'Église catholique, à propos du CIP:

La nécessité du bien général a voulu qu'au-dessus des commissions scolaires qui sont le rouage essentiel de notre système, il existe une autorité supérieure chargée de coordonner les efforts et d'exercer une tutelle nécessaire. Chez nous, l'Etat a jugé que cette tâche pourrait être exercée avec plus de stabilité et d'esprit de suite par un organisme auquel l'Etat aurait délégué la plus grande partie de ses pouvoirs et qu'il aurait soustrait aux contingences des régimes politiques. Ces considérations nous ont valu la formation du Conseil de l'Instruction Publique, qui constitue l'autorité supérieure en matière d'éducation. Bien qu'il exerce son action dans le même domaine que les commissions scolaires, le Conseil de l'Instruction Publique diffère grandement de celle-ci dans sa nature et ses attributions. Les commissions scolaires sont les mandataires des familles et elles jouissent de toute l'indépendance compatible avec les intérêts supérieurs de la nation. Le Conseil de l'Instruction Publique est le mandataire des droits de l'Etat. En le formant, celui-ci a tenu à faire partager la haute direction à l'Eglise en y appelant tous les évêques catholiques à y siéger ex-officio. [Filteau commet ici un anachronisme, puisque ce n'est qu'en 1875 que tous les évêques siégeront au conseil]. De là vint que les familles ne soient pas directement représentées dans ce Conseil. De là également cette prescription qui paraît singulière à plusieurs que «dans l'accomplissement de leurs devoirs, les membres sont sujets aux ordres et instructions que leur adresse le lieutenant-gouverneur-enconseil», principe que l'Etat a tenu à affirmer mais dont il ne s'est à peu près jamais prévalu. ${ }^{65}$

Une autre loi scolaire voyait le jour en 1856: l' «Acte pour faire de meilleures dispositions pour l'avancement de l'éducation supérieure ${ }^{66}$. Elle prévoyait la création de trois écoles normales confessionnelles, pour la formation des maîtres. Le caractère confessionnel du réseau scolaire s'accentuait, à cause de ces écoles normales (celles où l'enseignement se ferait en français seraient confiées au clergé), mais surtout parce que le CIP apprenait déjà à fonctionner sur une base confessionnelle.

64 Journal de l'Instruction Publique, 4,1 (juin 1860): 1.

65 Gérard Filteau, Les constantes historiques de notre système scolaire, 50 . On voit combien Filteau présente les choses de manière à appuyer ses présupposés idéologiques pro-cléricaux.

6619 Victoria, chapitre 54. 
A propos de ces lois, P.-J.-O. Chauveau écrivit: «La nouvelle législation remédie à la plupart des obstacles que j'avais signalés dans mon premier rapport, et fournit de plus les moyens d'opérer progressivement d'autres réformes qui donneront à notre système d'instruction publique toute l'efficacité désirable. ${ }^{67}$ Dès la promulgation des lois, le surintendant publiait une première circulaire (numéro 9) qui appelait l'attention des inspecteurs d'école, des commissaires et du public sur leurs dispositions. Une autre circulaire venait informer les mêmes personnes de la création des écoles normales, d'une caisse de retraite pour les instituteurs âgés ou devenus infirmes et de la publication du Journal de l'Instruction Publique qui commença sa carrière en $1856^{68}$.

Nous savons que l'article 93 de l'AABN vint, en quelque sorte, «immobiliser» le système scolaire. C'est en 1861 qu'une loi refondue mit de l'ordre, sans grande efficacité, dans les législations scolaires. Assez curieusement la loi refondue confiait encore aux commissaires l'embauche, la surveillance et le renvoi des maitres, la régie des écoles, le choix des manuels, des programmes, le classement des écoles (en établissements élémentaires, modèles et académiques). Et elle remettait au CIP le contrôle de la compétence des instituteurs, choix des livres, du matériel pédagogique et des programmes et le classement des écoles! Cette dernière institution avait sûrement la préséance ${ }^{69}$. Au niveau local, l'administration des écoles devait obéir aux règles du surintendant, ce dont s'assuraient les inspecteurs. La gestion du personnel était elle aussi, on l'a vu, orientée par l'équipe «surintendant-inspecteur». Et le surintendant pouvait retenir la part de l'octroi gouvernemental des arrondissements scolaires, lors d'infractions des commissaires ou des trésoriers, et les amener devant les tribunaux si la situation devenait plus sérieuse. A une époque où le Canada-Uni devait négocier le traité de réciprocité et construire le Grand-Tronc, l'État s'était doté d'un régime scolaire libéral, susceptible de permettre à la colonie de relever les défis politiques et économiques qui s'offraient à elle. Il devait assurer l'égalité «scolaire» des individus (toute relative, nous le savons), l'autre pôle de l'égalité devant la loi.

On a vu que l'Église catholique n'allait pas céder volontiers ses prérogatives, surtout au moment où Mgr Bourget donnait une impulsion toute nouvelle à son recrutement... et à ses ambitions. Et comme l'expliquait avec raison Nadia Fahmy-Eid ${ }^{70}$, la faiblesse des élites francophones (leur pouvoir politique étant tout relatif, leur pouvoir écono-

\footnotetext{
67 Pierre-Joseph-Olivier Chauveau, «Rapport sur l'Instruction publique dans le Bas-Canada pour l'année 1856», dans Journaux de l'Assemblée, 1857.

68 Ibid.

69 Un prochain article devrait mettre de l'ordre dans ces contradictions, en examinant l'exercice des responsabilités des différents niveaux administratifs.

70 Nadia Fahmy-Eid, loc . cit.
} 
mique négligeable) allait les obliger à une connivence coûteuse. Nous avons vu la faille s'élargir: le droit de dissidence, la présence de clercs dans les commissions scolaires, des commissions scolaires confessionnelles à Québec et à Montréal, des écoles normales confessionnelles, les décisions du CIP votées pour l'un ou l'autre des réseaux «confessionnels»... Cela n'affectait pas encore le caractère du réseau: l'État donnait une place à l'Église, il pouvait tout aussi bien la lui retirer... s'il l'osait. Mais l'Église avançait ses pions, faisait pièce aux libéraux et se liait pour «services rendus» au Parti conservateur au pouvoir pour ce demi-siècle. Elle ne pouvait encore aller trop loin: le Canada-Uni comptait une population majoritairement anglophone et, au HautCanada, le Parti libéral volontiers antipapiste établissait un pouvoir qui allait durer jusqu'à la fin du siècle.

Cependant elle allait chaudement appuyer le projet confédératif et, une fois acquise la nouvelle constitution, se lancer à l'assaut de l'État déjà exsangue ${ }^{71}$ du Québec: dans une société majoritairement catholique et française, elle allait assumer certaines des responsabilités que lui laissait la constitution. Sa victoire survint rapidement: la réforme du CIP créait deux réseaux scolaires confessionnels parallèles en 1869 , celle de 1875 lui livrait le réseau catholique et faisait disparaître le ministère de l'Instruction publique. Peut-on croire que le système scolaire avait gardé, après cela, des caractères libéraux.

71 Les pouvoirs confiés au gouvernement central, le droit de celui-ci de défaire des lois provinciales, de voter éventuellement, en ce qui concerne spécifiquement les écoles, des lois réparatrices, laissaient peu de latitude au gouvernement provincial. Abandonner une partie des prérogatives de cet État à l'épiscopat n'était pas pour améliorer les choses. 